\title{
MODELING FAMILIES OF L-FUNCTIONS
}

\author{
DAVID W FARMER
}

\begin{abstract}
We discuss the idea of a "family of $L$-functions" and describe various methods which have been used to make predictions about $L$-function families. The methods involve a mixture of random matrix theory and heuristics from number theory. Particular attention is paid to families of elliptic curve $L$-functions. We describe two random matrix models for elliptic curve families: the Independent Model and the Interaction Model.
\end{abstract}

\section{INTRODUCTION}

Using ensembles of random matrices to model the statistical properties of a family of $L$ functions has led to a wealth of interesting conjectures and results in number theory. In this paper we survey recent results in the hopes of conveying our best current answers to these questions:

(1) What is a family of $L$-functions?

(2) How do we model a family of $L$-functions?

(3) What properties of the family can the model predict?

In the remainder of this section we briefly review some commonly studied families and describe some of the properties which have been modeled using ideas from random matrix theory. In Section 2 we provide a definition of "family of $L$-functions" which has been successful in permitting precise conjectures, and we briefly describe how to model such a family. In Section 3 we discuss families of elliptic curve $L$-functions and show that there is an additional subtlety which requires us to slightly broaden the class of random matrix models we use. Then in Section 4 we discuss how to go beyond the leading-order terms which random matrix theory can model, and how one can avoid using random matrix theory when modeling a family of $L$-functions.

If you only care about elliptic curves and their $L$-functions you can safely skip to Section 3 .

I thank Brian Conrey, Nina Snaith, Matt Young, and Steven J. Miller for many helpful conversations.

1.1. A quick history of families. The idea that collectively the zeros of a single $L$-function behave in a manner that can be modeled statistically started with Montgomery's [Mon] work on the pair correlation of zeros of the zeta-function. Combined with the large-scale numerical calculations of Odlyzko Odl, this provided convincing evidence that, to leading order, the local statistics of the zeros of the zeta-function, suitably rescaled, were the same as those of large random unitary matrices.

Work supported by the American Institute of Mathematics and by the Focused Research Group grant (0244660) from the NSF. This paper is an expanded version of a talk given at the workshop "Random Matrix Theory and Elliptic Curves" held at the Isaac Newton Institute, February 2004. 
A similar collective behavior was noted long ago and is termed the " $q$-analogue" for Dirichlet $L$-functions. That is, results about all Dirichlet $L$-functions $\bmod q$ look just like results for the Riemann $\zeta$-function in $t$-aspect. For example, the formulas for moments of $\left|\zeta\left(\frac{1}{2}+i t\right)\right|$ and $|L(s, \chi)|$ are identical but for replacing $t$ by $q$. Another early example of collective behavior is the pair correlation of zeros of quadratic Dirichlet $L$-functions [OS1. Clearly something interesting is going on.

The idea of a family of $L$-functions with an associated symmetry type began with the work of Katz and Sarnak [KSa]. They consider families of function field $L$-functions, where in this case a "family" of $L$-functions is the set of $L$-functions associated to a set of curves having certain properties. Here the collection of curves must be "natural" in the sense that the monodromy group of the family ties it all together. They show that to leading order the statistics of the (normalized) zeros of these $L$-functions, when averaged over the family, are the same as the statistics of the (normalized) eigenvalues of random matrices chosen from a classical compact group. Here the matrices are chosen uniformly with respect to Haar measure, and the size of the matrices scales with the conductor of the $L$-function.

For global $L$-functions there does not (yet?) exist an analogue of "monodromy", but it still has been found that to naturally occurring families one can associate a classical group of matrices. The zeros of the $L$-functions have, to leading order, the same statistics as the eigenvalues of a randomly chosen matrix from the group. And the appropriately rescaled critical values of the $L$-functions have, to leading order, the same distribution as the "critical values" of the characteristic polynomials of the matrices from the group, chosen uniformly with respect to Haar measure. The correspondence between the matrices and the $L$-functions involves equating the eigenvalue spacing with the zero spacing, or equivalently setting the matrix size equal to the conductor of the $L$-function. See Section 2.4 for more details.

After the work of Katz and Sarnak there quickly appeared many examples of $L$-functions families behaving in a manner predicted by random matrix theory. Some of the families considered were: $L$-functions associated to holomorphic cusp forms (in either weight or level aspect); Dirichlet $L$-functions (either all or quadratic); and various twists or symmetric powers of $L$-functions. Low-lying zeros were considered by Iwaniec, Luo, and Sarnak [ILS], Rubinstein [Rub], Özlük and Snyder [OS2], and others. Moments were considered by Iwaniec and Sarnak [IS], Kowalski, Michel, and VanderKam [KMV], Soundararajan [S], and others.

There is an important distinction between the predictions for zeros of $L$-functions and the predictions for moments. For the zeros there is a natural way to normalize: rescale so that the average spacing is 1 . This rescaling involves the conductor of the $L$-function and the degree of the characteristic polynomial, and this is the source of the principle that one chooses the size of the random matrix to equal the (logarithm of the) conductor of the $L$ function. With these normalizations one obtains accurate predictions for the leading-order behavior of statistics of zeros of $L$-functions. However, in addition to its zeros a polynomial is determined by an overall scale factor. So one may hope to use the characteristic polynomials to model the $L$-functions, but there will be a correction factor that does not come from random matrix theory. The use of the characteristic polynomial to model the $L$-function was begun by Keating and Snaith KS1. The first situation which was well understood is the moments of $L$-functions, for which there are explicit predictions for the arithmetic scale factor. See CG, CF, KS2]. 


\section{What IS A FAMiLY?}

There does not yet exist an adequate definition of "family of $L$-functions". An attempt is made in CFKRS to define a family axiomatically, and we will describe that definition here. In that definition the axioms are chosen so that it is possible to produce a plausible conjecture for the critical moments of the family.

Complete details and many examples are in [CFKRS, so we will just highlight the key features of that definition of a family. The main idea is that one starts with a fixed $L$-function and a family of "characters", and the family of $L$-functions is produced by twisting the fixed $L$-function by the family of characters. Note that here the term character is used to cover more general classes of functions than just Dirichlet characters.

2.1. $L$-functions. We wish to define a "family of $L$-functions", so first we have to give the definition we will use for " $L$-function". The definition of an $L$-function which we give below is slightly different than what is known as the "Selberg class," but it is conjectured that the two are in fact equal.

Let $s=\sigma+i t$ with $\sigma$ and $t$ real. An $L$-function is a Dirichlet series

$$
L(s)=\sum_{n=1}^{\infty} \frac{a_{n}}{n^{s}},
$$

with $a_{n}=O_{\varepsilon}\left(n^{\varepsilon}\right)$ for every $\varepsilon>0$, which has three additional properties.

Analytic continuation: $L(s)$ continues to a meromorphic function of finite order with at most finitely many poles, and all poles are located on the $\sigma=1$ line.

Functional equation: There is a number $\varepsilon$ with $|\varepsilon|=1$, and a function $\gamma_{L}(s)$ of the form

$$
\gamma_{L}(s)=P(s) Q^{s} \prod_{j=1}^{w} \Gamma\left(\frac{1}{2} s+\mu_{j}\right),
$$

where $Q>0, \Re \mu_{j} \geq 0$, and $P$ is a polynomial whose only zeros in $\sigma>0$ are at the poles of $L(s)$, such that

$$
\xi_{L}(s):=\gamma_{L}(s) L(s)
$$

is entire, and

$$
\xi_{L}(s)=\varepsilon \overline{\xi_{L}}(1-s),
$$

where $\overline{\xi_{L}}(s):=\overline{\xi_{L}(\bar{s})}$ and $\bar{s}$ denotes the complex conjugate of $s$.

The number $w$ is called the degree of the $L$-function. That number will also appear in the Euler product.

Euler product: For $\sigma>1$ we have

$$
L(s)=\prod_{p} \prod_{j=1}^{w}\left(1-\gamma_{p, j} p^{-s}\right)^{-1}
$$

where the product is over the primes $p$, and each $\left|\gamma_{p, j}\right|$ equals 1 or 0 .

Note that $L(s) \equiv 1$ is the only constant $L$-function, the set of $L$-functions is closed under products, and if $L(s)$ is an $L$-function then so is $L(s+i y)$ for any real $y$. An $L$-function is called primitive if it cannot be written as a nontrivial product of $L$-functions. Throughout 
this paper we assume all $L$-functions are primitive, although we usually omit the word "primitive."

Conductor: Associated to an $L$-function is its conductor, a number which measures the "size" of the $L$-function. The paper CFKRS introduced a refined notion of conductor which, to leading order, is the logarithm of the usual notion of conductor. The refined conductor is necessary in order to have any hope of conjecturing the full main term in a general mean value of the $L$-function. Write the functional equation in asymmetric form:

$$
L(s)=\varepsilon X_{L}(s) \bar{L}(1-s),
$$

where $X_{L}(s)=\frac{\overline{\gamma_{L}}(1-s)}{\gamma_{L}(s)}$. Then the refined conductor of $L(s)$, denoted $c(L)$, is given by $c(L)=\left|X_{L}^{\prime}\left(\frac{1}{2}\right)\right|$.

2.2. Families of characters. By a family of characters we mean a collection of arithmetic functions $\mathcal{F}$, where each $f \in \mathcal{F}$ is a sequence $f(1)=1, f(2)=a_{2, f}, f(3)=a_{3, f}, \ldots$ whose generating function

$$
L_{f}(s)=\sum_{n=1}^{\infty} \frac{a_{n, f}}{n^{s}}=\prod_{p} \prod_{j=1}^{v}\left(1-\beta_{p, j} p^{-s}\right)^{-1}
$$

is a (primitive) $L$-function such that the collection $\left\{L_{f}: f \in \mathcal{F}\right\}$ has some nice properties. If we order the $L$-functions $L_{f}$ by conductor $c(f)$, then the data $\left\{Q ; \mu_{1}, \ldots, \mu_{w}\right\}$ in the functional equation of $L_{f}$ should be monotonic functions of the conductor, and the counting function $M(X):=\#\{f \in \mathcal{F} \mid c(f) \leq X\}$ should be nice. The final condition on the family of characters is the existence of an orthogonality relation among the $f \in \mathcal{F}$. Specifically, we require that if $m_{1}, \ldots, m_{k}$ are integers then the average

$$
\delta_{\ell}\left(m_{1}, \ldots, m_{k}\right):=\lim _{X \rightarrow \infty} M(X)^{-1} \sum_{\substack{f \in \mathcal{F} \\ c(f) \leq X}} f\left(m_{1}\right) \ldots f\left(m_{\ell}\right) \overline{f\left(m_{\ell+1}\right) \ldots f\left(m_{k}\right)}
$$

exist and be multiplicative. That is, if $\left(m_{1} m_{2} \ldots m_{k}, n_{1} n_{2} \ldots n_{k}\right)=1$, then

$$
\delta_{\ell}\left(m_{1} n_{1}, m_{2} n_{2}, \ldots, m_{k} n_{k}\right)=\delta_{\ell}\left(m_{1}, \ldots, m_{k}\right) \delta_{\ell}\left(n_{1}, \ldots, n_{k}\right) .
$$

See Section 3.1 of [CFKRS] for more details.

2.3. Families of $L$-functions. Now we create a family of $L$-functions by starting with a fixed $L$-function

$$
L_{g}(s)=\sum_{n=1}^{\infty} \frac{a_{n, g}}{n^{s}}=\prod_{p} \prod_{j=1}^{w}\left(1-\gamma_{p, j} p^{-s}\right)^{-1} .
$$

Then the elements of our $L$-function family $\mathcal{L}(\mathcal{F})$ are the Rankin-Selberg convolutions

$$
\begin{aligned}
\mathcal{L}(s, f)=L_{f \times g}(s) & =\prod_{p} \prod_{i=1}^{v} \prod_{j=1}^{w}\left(1-\beta_{p, i} \gamma_{p, j} p^{-s}\right)^{-1} \\
& =\sum_{n=1}^{\infty} \frac{a_{n, f \times g}}{n^{s}} .
\end{aligned}
$$


(There may be some issues with the local factors at the bad primes). Note that if $w=1$ or $v=1$ then

$$
\mathcal{L}(s, f)=\sum_{n=1}^{\infty} \frac{a_{n, f} a_{n, g}}{n^{s}} .
$$

And in particular if $L_{g}$ is the Riemann zeta-function, then $\mathcal{L}(s, f)=L_{f}(s)$.

The point of this definition of "family" is that the axioms provide the necessary ingredients to apply the recipe in CFKRS to conjecture the full main term in the shifted $K$ th moment

$$
M(X)^{-1} \sum_{c(f) \leq X} \prod_{1 \leq k \leq K} L\left(\frac{1}{2}+\alpha_{k}\right)
$$

or more generally a shifted ratio CFZ

$$
M(X)^{-1} \sum_{c(f) \leq X} \prod_{1 \leq k \leq K} \frac{L\left(\frac{1}{2}+\alpha_{k}\right)}{L\left(\frac{1}{2}+\delta_{k}\right)} .
$$

Having such a mean value is sufficient to conjecture just about anything you would like to know about the zeros and the value distribution of the $L$-function. See CS for examples.

Note that some families are unions of increasingly large pieces having the same conductor. Examples are the Dirichlet $L$-functions and the $L$-functions associated to holomorphic cusp forms (in either weight or level aspect). For those families it is believed that the heuristics for moments will produce a reasonable conjecture for the average over a fixed (large) conductor.

Although this definition of "family" is useful for certain applications, it lacks the concreteness of the function field case. In particular, there does not yet exist an analogue of monodromy for such a family, and computing the symmetry type of the family is not straightforward. We discuss this in the next section.

2.4. Modeling a family of $L$-functions. Given a family of $L$-functions one can ask questions about its value distribution or about the distribution of its zeros. In most cases current technology is not sufficient to answer the interesting questions, so the next hope is to find a plausible conjecture. Only recently have such conjectures been found, and the new ingredient is to use random matrices to model the family of $L$-functions.

The idea is to associate a classical compact group, $U(N), S p(2 N), O(N), S O(2 N)$, or $S O(2 N+1)$, to the family. The local statistics of the eigenvalues should agree, to leading order, with the corresponding local statistics of the zeros of the $L$-functions. And, to leading order and after compensating by an arithmetic constant, the value distribution of the characteristic polynomial

$$
\Lambda(z)=\Lambda_{A}(z)=\operatorname{det}\left(I-A^{*} z\right)=\prod_{n=1}^{N}\left(1-z e^{-i \theta_{n}}\right)
$$

near the point $z=1$ should agree with the value distribution of the $L$-functions near the critical point. Here $A$ is an $N \times N$ unitary matrix $A$ and $A^{*}$ is the Hermitian conjugate of $A$, so the eigenvalues of $A$ lie on the unit circle and are denoted by $e^{i \theta_{n}}$. 
In the above correspondence the size of the matrix is set equal to the conductor of the $L$-function. (Actually, to an integer close to the conductor, but to leading order such discrepancies do not matter). To see why this is a natural choice, consider the functional equation satisfied by the characteristic polynomial:

$$
\Lambda_{A}(z)=(-1)^{N} \operatorname{det}(A) z^{N} \Lambda_{A^{*}}\left(z^{-1}\right) .
$$

If we identify $(-1)^{N} \operatorname{det}(A)$ with $\varepsilon$ and $z^{N}$ with $X_{L}(s)$ then we have a perfect correspondence between the functional equations of $\Lambda_{A}(z)$ and $L(s)$, the unit circle playing the role of the critical line and $z=1$ the critical point. Just as for $L$-functions, we define the conductor as $\frac{d}{d z} z^{N}$ evaluated at the critical point, so $N$ is the conductor. Note that identifying conductors is equivalent to equating the average spacing between the zeros. Values near the critical point are modeled using the correspondence $\Lambda\left(e^{-z}\right) \leftrightarrow L\left(\frac{1}{2}+z\right)$.

It remains to identify the matrix group which corresponds to the family. From the functional equation it is almost possible to determine the group: the only ambiguity is to distinguish between $S O(2 N)$ and $S p(2 N)$. At one time it was thought that this case could be easily resolved because $S O(2 N)$ families always arise as "half" of a larger family, the other half being modeled by $S O(2 N+1)$. On the other hand $S p(2 N)$ families do not have such a "partner". A counterexample to that hope is described in [MD2]. But even if that approach were viable, it is unsatisfactory because it relies on the fact that the symmetry type can be found among a small list of possibilities. Fortunately, there are other methods. One possibility is to compute the 1- and 2-level densities of the family. This usually can be done rigorously for functions with small support, and this is sufficient to distinguish among the classical compact groups. But again we are relying on the fact that the symmetry type can be found on a short list. That objection can be overcome if one can conjecture the level densities in the full range, but that can be quite difficult in practice. Another possibility is to use the recipe in CFKRS to conjecture the moments of the family. This unambiguously identifies the group, and it also can tell you if the family is not modeled by one of those groups. Unfortunately, it is not clear that the recipe in CFKRS can be applied to all interesting families of elliptic curve $L$-functions, such as the family $\mathcal{F}_{3}$ given in (3.6) .

2.5. Summary of modeling. Just to be pedantic, we note the following answers to the questions posed at the beginning of Section 1 .

(1) A family of $L$-functions is a set of $L$-functions, ordered by conductor, which is built in a particular way from a family of characters. The counting function of the family should be nice, and the data in the functional equation should be monotonic functions of the conductor.

(2) A family is modeled by associating to it a classical compact matrix group. The specific compact group can usually be determined by computing the level densities of the low-lying zeros of the family, or by conjecturing the moments of the family. The size of the matrices scales with the (logarithmic) conductor of the $L$-functions.

(3) To leading order the rescaled local zero statistics of the family are the same as the rescaled local eigenvalue statistics of the group. To leading order the critical moments of the family equal the critical moments of the characteristic polynomials, up to a multiplicative arithmetic constant. For the family we average over the $L$-functions of conductor less than $X$, and then let $X \rightarrow \infty$. For the matrix groups the averages are with respect to Haar measure. 
The modeling described above will produce leading order asymptotics. To make more precise predictions requires other methods, which are described in Section 4 .

\section{Elliptic CURVE FAMilies}

For the remainder of the paper, $E$ is an elliptic curve over $\mathbb{Q}$, with root number $w_{E}= \pm 1$, and $L(s, E)$ is the $L$-function associated to $E$ normalized so that $s=\frac{1}{2}$ is the critical point. If $F$ is any family of elliptic curves we write $F=F^{+} \cup F^{-}$where $F^{+}$or $F^{-}$, respectively, are the curves $E \in F$ with $w_{E}=+1$ or $w_{E}=-1$. We write $E_{a, b}$ for the curve $y^{2}=x^{3}+a x+b$.

Most of the information in this section can be found in recent papers by Steven J. Miller and Eduaro Dueñez [M], IMD1, IMD2, Nina Snaith [Sn1, Sn2] and Matthew Young YY1, Y2, $\mathrm{Y} 3$. The author of this paper is just trying to convey the current understanding of the relationship between families of elliptic curve $L$-functions and random matrix theory: he makes no claim to any of the ideas presented here.

3.1. Families with a given rank. The following question is not well posed:

What is the correct random matrix model for the L-functions of a family of elliptic curves having a prescribed rank $r$ ?

The question is not well posed because there are (at least) two reasonable models, both of which seem to be appropriate for certain families of elliptic curves. We will first examine the simplest case of rank $r=1$.

Consider the following families of rational elliptic curves:

$$
\mathcal{F}_{1}(X)=\left\{E_{a, b}:|a| \leq X^{\frac{1}{3}},|b| \leq X^{\frac{1}{2}}\right\}
$$

and

$$
\mathcal{F}_{2}(X)=\left\{E_{a, b^{2}}:|a| \leq X^{\frac{1}{3}}, b^{2} \leq X^{\frac{1}{2}}\right\} .
$$

Note that the point $(0, b)$ on $E_{a, b^{2}}$ almost always has infinite order, so almost all of the curves in $\mathcal{F}_{2}$ have rank at least 1.

Let's consider $\mathcal{F}_{1}^{-}$and $\mathcal{F}_{2}^{-}$. In both families we have $L\left(\frac{1}{2}, E\right)=0$ because $w_{E}=-1$. However, for $E \in \mathcal{F}_{2}^{-}$we could have said $L\left(\frac{1}{2}, E\right)=0$ because $\operatorname{rank}(E) \geq 1$. The fact that the zero at $L\left(\frac{1}{2}, E\right)$ for $E \in \mathcal{F}_{2}$ was constructed, instead of just arising from parity considerations, has a profound influence on the behavior of the $L$-function near the critical point.

To understand the influence of the critical zero, we first consider the distribution of $L^{\prime}\left(\frac{1}{2}, E\right)$, which assuming standard conjectures is nonzero for almost all curves in our $w_{E}=-1$ families. We have the following conjectures from [Y2]:

$$
\frac{1}{\left|\mathcal{F}_{1}^{-}(X)\right|} \sum_{E \in \mathcal{F}_{1}^{-}(X)} L^{\prime}\left(\frac{1}{2}, E\right)^{k} \sim c_{1}(k)(\log X)^{k(k+1) / 2}
$$

while

$$
\frac{1}{\left|\mathcal{F}_{2}^{-}(X)\right|} \sum_{E \in \mathcal{F}_{2}^{-}(X)} L^{\prime}\left(\frac{1}{2}, E\right)^{k} \sim c_{2}(k)(\log X)^{k(k-1) / 2}
$$


As the formulas show, the behavior at the critical point is different for the two families, even though both families could be described as "a rank 1 family of elliptic curves." In particular, we see that the derivative $L^{\prime}\left(\frac{1}{2}, E\right)$ tends to be smaller for $E \in \mathcal{F}_{2}^{-}$. This can be explained by the tendency for the low-lying zeros of $L(s, E)$ to be closer to the critical point for $E \in \mathcal{F}_{2}^{-}$. That is, $\mathcal{F}_{2}^{-}$should have more low-lying zeros, which will cause the $L$-function to stay small near the critical point, and so its derivative will also be small. To make this idea precise we consider the 1-level density of the zeros.

Let $0<\gamma_{E, 1} \leq \gamma_{E, 2} \leq \gamma_{E, 3} \leq \cdots$ denote the imaginary parts of the zeros of $L(s, E)$ in the upper half of the critical strip. Note that we have omitted the zero(s) at the critical point. The one-level density of the family $F(X)$ is defined to be the function $W_{1}$ which satisfies

$$
\frac{1}{|F(X)|} \sum_{E \in F(X)} \sum_{j} \phi\left(\gamma_{E, j}\right) \sim \int \phi(t) W_{1}(t) d t
$$

as $X \rightarrow \infty$, for nice functions $\phi$. That is, $W_{1}$ measures the density of the zeros of the family.

The observation about the relative size of $L^{\prime}\left(\frac{1}{2}, E\right)$ can be restated as: the one-level density for the family $\mathcal{F}_{2}^{-}$should be more concentrated near 0 than the one-level density for the family $\mathcal{F}_{1}^{-}$. By using random matrix theory and some other ideas we explain below, it is possible to produce a precise conjecture for the one-level densities of these families. These are given in Figure 3.1. The functions are rescaled so that the average spacing between zeros is 1 . In the next section we explain where those conjectures came from.
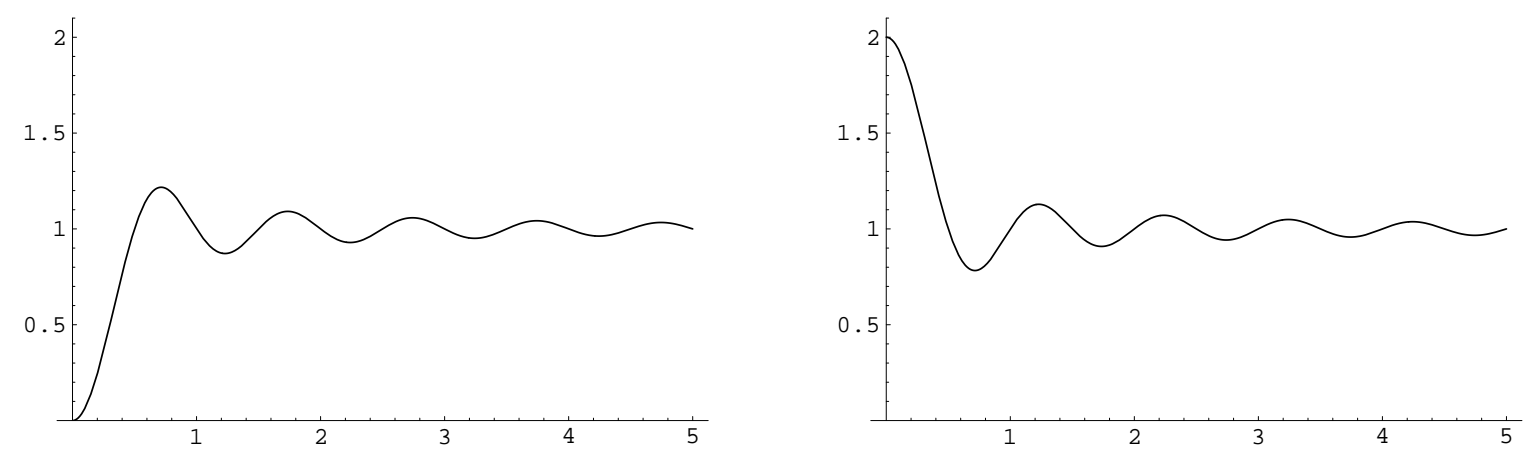

FigurE 3.1. Conjectured one-level density of the noncritical $L$-function zeros of the family $\mathcal{F}_{1}^{-}$(left) and $\mathcal{F}_{2}^{-}$(right).

3.2. Two models for two kinds of families. The plots in Figure 3.1 are familiar. The plot on the left is the rescaled one-level density of the eigenvalues of matrices from the group $S O(2 N+1)$, in the limit as $N \rightarrow \infty$. The plot on the right is the rescaled one-level density of the eigenvalues of matrices from the group $S O(2 N)$, in the limit as $N \rightarrow \infty$. Given those plot, what are our models for the two families?

Consider the following ways to make a polynomial $f(z)$ which has real coefficients, all its zeros on the unit circle, and (almost surely a simple) zero at $z=1$ :

- The characteristic polynomial of a matrix in $S O(2 N+1)$

- $(z-1)$ times the characteristic polynomial of a matrix in $S O(2 N)$ 
It should be clear that those two examples will have the one-level densities pictured in Figure 3.1. These examples are the simplest cases of the two most commonly studied higher rank families of elliptic curve $L$-functions, which we now describe.

Suppose $E_{T}$ is a curve $y^{2}=x^{3}+a(T) x+b(T)$ of rank $r$ over $\mathbb{Q}(T)$. Consider the following two families of rational elliptic curves:

$$
\mathcal{F}_{3}(X)=\left\{E_{a, b}:|a| \leq X^{\frac{1}{2}},|b| \leq X^{\frac{1}{3}}, \operatorname{rank}(E) \geq r\right\}
$$

and

$$
\mathcal{F}_{4}(X)=\left\{E_{t}:|a(t)| \leq X^{\frac{1}{2}},|b(t)| \leq X^{\frac{1}{3}}, t \in \mathbb{N}\right\} .
$$

As in our rank 1 example, we have $\mathcal{F}_{4}(X) \subset \mathcal{F}_{3}(X)$. Again we consider the subfamilies according to the sign of $w_{E}$. Let $F$ be either of the above rank $r$ families. If $r$ is odd then almost all the curves in $F^{-}$have rank $r$, and almost all the curves in $F^{+}$have rank $r+1$. If $r$ is even then the $F^{+}$curves have rank $r$ and the $F^{-}$curves have rank $r+1$. It is conjectured that $\mathcal{F}_{4}^{+}$and $\mathcal{F}_{4}^{-}$are approximately the same size provided $E$ has at least one place of multiplicative reduction. See [H]. If $r$ is even then it is possible that $\mathcal{F}_{3}^{+}$and $\mathcal{F}_{3}^{-}$ are approximately the same size.

We describe the two models which are believed to correspond to these families. The names for these models was coined by Steven J. Miller.

\subsection{Selecting to have zeros. The Interaction Model}

We are modeling a family that arises by restricting a much larger family to a subfamily having at least $r$ zeros at the critical point. A matrix model for this family can be described as follows: start with $S O(M)$ where $M=2 N$ or $2 N+1$ depending on whether we are modeling $\mathcal{F}_{3}^{+}$or $\mathcal{F}_{3}^{-}$, and restrict to those matrices having 1 as an eigenvalue of multiplicity at least $r$. That is, you take matrices in $S O(M)$ and drag $r$ zeros to the critical point.

There are some bad things about this model. First, it is a set of matrices, but it is not a group. And while it is a perfectly well-defined set, it is a measure zero subset of $S O(M)$, so there is no canonical way to restrict Haar measure to it.

One solution, which has been analyzed by Snaith [Sn1] and Dueñez [D, MD1] is to first restrict to those matrices which have $r$ eigenvalues in $[-\varepsilon, \varepsilon]$, and then let $\varepsilon \rightarrow 0$. The resulting measure is the same as one obtains by taking Haar measure on $S O(M)$, formally substituting $\theta=0$ for $r$ of the eigenvalues, and then omitting those terms which vanish identically. With the eigenvalues given by $e^{i \theta_{j}}$, the induced measure on that set is

$$
C(M, r) \prod_{j=1}^{M}\left(1-\cos \theta_{j}\right)^{r} \prod_{1 \leq j<k \leq M}\left(\cos \theta_{j}-\cos \theta_{k}\right)^{2} d \theta_{1} \cdots d \theta_{M}
$$

where $C(M, r)$ is a normalization constant.

It is instructive to look at the one-level density for such matrices. The one-level density is given by Sn1, MD1.

$$
\frac{\pi^{2}}{2} \theta\left(J_{r-\frac{3}{2}}^{2}(\theta \pi)+J_{r-\frac{1}{2}}^{2}(\theta \pi)-\frac{2 r-1}{\theta \pi} J_{r-\frac{1}{2}}(\theta \pi) J_{r-\frac{3}{2}}(\theta \pi)\right) .
$$

There is some numerical evidence [MD1] that this model is accurate. For this model it is possible to compute the critical moments of the characteristic polynomials [Sn1], but it does 


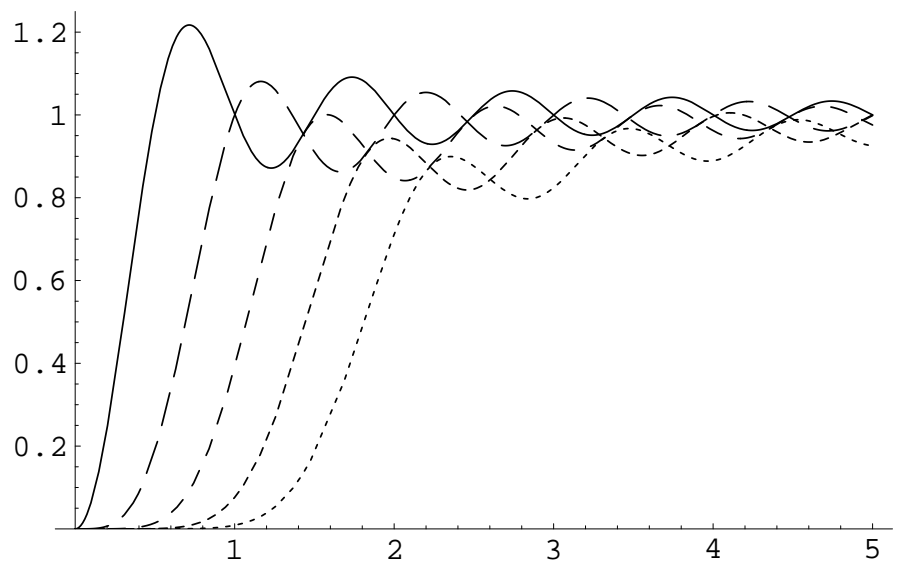

Figure 3.2. One-level density for $S O(M)$, restricted to have exactly $r$ eigenvalues at $\theta=0$, for $r=1,2,3,4,5$.

not seem that all the ingredients are available to use the recipe in CFKRS to conjecture the moments of the family.

Note that when $r=0$ we recover $S O(2 N)$ and when $r=1$ we have $S O(2 N+1)$.

\subsection{Imposing zeros. The Independent Model}

We are modeling a family that has an $r$ th order zero at the origin which arises from an explicit construction. A model for this situation can be found by assuming that the extra critical zeros are just inserted at the critical point, and all the other zeros ignore them. That is, start with a matrix in $S O(M)$ where $M=2 N-r$ or $2 N+1-r$ depending on the parity of $r$ and the sign of $w_{E}$. Then the polynomial which models the $L$-function is the characteristic polynomial of the matrix, multiplied by $(x-1)^{r}$. The one-level density only depends on the parity of $r$ and the sign of $w_{E}$, and will be one of the functions shown in Figure 3.1

One can phrase the model strictly in terms of matrices by saying that the model is given by the group

$$
\left(\begin{array}{cc}
I_{r \times r} & \\
& S O(M)
\end{array}\right)
$$

where $I_{r \times r}$ is the $r \times r$ identity matrix and $M=2 N-r$ or $2 N+1-r$ is chosen according to the parity of $r$ and the sign of the functional equation.

There is numerical evidence [MD1, level density calculations [M, MD1, Si, Y1] and conjectures for moments $\mathrm{Y} 2$ that this model gives accurate predictions for some specific families.

3.5. Some issues. It is worth repeating that the above models, even if they are correct, are only intended to capture leading-term asymptotics. Computer experiments [MD1 find that the low lying zeros of the family $\mathcal{F}_{4}$ exhibit some anomalous behavior which presumably will disappear when larger examples are computed. It is entirely possible that family $\mathcal{F}_{3}$ is a union of families of the form $\mathcal{F}_{4}$, and this may contribute to a bias in those numerics. In families of type $\mathcal{F}_{4}$ the generators of the set of rational points have very small height, and this may also introduce a bias. That is, the heights of the generators are on the order of the 
logarithm of the conductor, while it is more typical to have the heights as large as a power of the conductor. See Silverman [Si2], Chapter 10, for a discussion of heights of generators.

It is possible that more accurate predictions of the one-level density (using methods described in the next section) will show better agreement with the data. Those methods are also able to give extremely precise predictions for the moments of the $L$-functions $[Y 2$, and these give support to the models.

It is not clear that these families of elliptic curves give rise to families of $L$-functions as described in Section 2. For the purpose of conjecturing the moments of the family (which is why that definition of 'family' was developed), the key property is the orthogonality relation (2.8). For specific families of type $\mathcal{F}_{4}$ it should be possible to evaluate such sums. The result is likely to be quite complicated, as in [Y2. A subtle problem is that the parameter $X$ in the elliptic curve families is approximately the discriminant, not the conductor. By Szpiro's conjecture the logarithm of the discriminant is within a factor of 6 of the logarithm of the conductor, so it is possible that ordering by discriminant is almost as good as ordering by conductor. It seems reasonable to model by setting $N$, the size of the matrix, equal to $\log X$, since what else would you choose? If that choice is correct it suggests that $X$ is close to the discriminant most of the time. This has been shown for some families [Y1].

One can cook up an elliptic curve family which presumably is a hybrid of the models described here: take a rank $r$ family of type $\mathcal{F}_{4}$ and restrict to those curves having rank at least $r+2$. If one makes the reasonable assumption that the "extra" zeros created by this process do not interact with the original $r$ zeros imposed at the critical point, then one can use methods similar to CKRS to predict how many curves are in the restricted family. I am not advocating a reckless proliferation of elliptic curve models, but merely noting that even if the two models described here are correct and can be refined to predict lower order terms, they may not cover all families of interest.

In Figure 3.3 one can see that if $r$ is large then you are unlikely to find noncritical zeros close to the critical point. The name "repulsion" has been given to this phenomenon. The logic behind the name is that the "lowest zero" is further from the critical point than it would be if there were not a multiple critical zero. Unfortunately, the "lowest zero" is not a well defined object. If you drag the lowest zero to the critical point then the other zeros follow it toward the critical point, and at the moment you increase the order of the critical zero there becomes a new "lowest zero". If there is an $r$ th order critical zero and you count zeros correctly, then the "lowest zero" is actually the $(r+1)$ st zero, and it is likely to be closer to the critical point than a typical $(r+1)$ st zero. In other words, the word "attraction" more accurately describes the situation! There is no reason to change the current terminology, but keep in mind that in the model where one restricts to those matrices having multiple eigenvalues at 1 , the other eigenvalues have actually moved towards the critical point.

\section{REFined MODELING}

Random matrix theory is useful for making leading-order asymptotic predictions about families of $L$-function. To understand the finer behavior of the family one must use heuristic techniques from number theory.

There are two main refinements to the leading term asymptotics. First, $L$-function families are ordered by conductor, and we use the conductor to determine the appropriate size 
matrices for our model. There will be a discrepancy between the limiting behavior for large matrices and the behavior for finite size matrices. For some quantities, such as the nearest neighbor spacing of the zeros/eigenvalues, it is difficult to see the difference between the asymptotics of the distribution and the distribution for moderate size matrices. For other quantities, such as the value distribution, for any computable range there is a notable difference between the limiting quantity and the values that can be computed. See Keating and Snaith [KS1, for a spectacular example concerning the value distribution of $\Re \log \zeta\left(\frac{1}{2}+i t\right)$. By a theorem of Selberg that is Gaussian in the limit $t \rightarrow \infty$, but for finite $t$ it differs from a Gaussian in the same way as the characteristic polynomial of an appropriately sized random unitary matrix.

The second issue is the fact that there are lower order terms, and the lower order terms for $L$-functions involve arithmetic factors, while the lower order terms of random matrices do not. Thus, the general shape of expressions from random matrix theory can reveal what to expect for $L$-functions, but the arithmetic "correction terms" must be determined in some other manner. For zero spacings there are no arithmetic corrections in the leading order terms. For moments of $L$-functions the leading order correction terms are fairly straightforward to determine [CG, CF, KS2]. Just about everything else is quite subtle and one needs sophisticated number-theoretic methods in order to make sensible conjectures.

For moments of $L$-functions such conjectures are covered in detail in CFKRS. Matt Young Y2 used these heuristics to compute the full main term for various families of elliptic curve $L$-functions. Those conjectures give (3.3) and (3.4) as special cases. Thus, the heuristics appear to correctly handle a variety of interesting families. (For the family $\mathcal{F}_{3}$ in (3.6) our current understanding of the distribution of the coefficients $a_{p}$ does not seem adequate to conjecture the moments of the family. Even finding the leading order arithmetic factor seems difficult in this case. However, random matrix calculations [Sn1] predict the general shape of the moments.)

For quantities involving zero statistics, moments are insufficient and one needs averages of ratios of the $L$-functions. This is addressed in [CFZ]. For example, the expected value of the ratio

$$
\frac{L\left(\frac{1}{2}+\alpha, f\right)}{L\left(\frac{1}{2}+\beta, f\right)}
$$

is sufficient to determine the one-level density of the family $L(s, f)$, including the lower order correction terms due to arithmetic effects. See [CS] for many examples. It should be possible to use these methods to conjecture the ratio (4.1) for various higher rank elliptic curve families, and thus give a precise conjecture for the one-level density. As of this writing this has not been done, but probably it will have been done by the time this paper appears in print.

In summary, by choosing the matrix group and the size of the matrices appropriately, and with the appropriate arithmetic correction factor, a family of $L$-functions can be modeled by the characteristic polynomials of a collection of matrices. The example of elliptic curve $L$-functions where the elliptic curves are selected to have large rank shows that the collection of matrices may not be a group. In order to capture the lower order terms one must use heuristics from number theory which do not explicitly involve random matrix theory. Those 
heuristics also recover the leading order behavior which previously required random matrix theory.

\section{REFERENCES}

[CG] J. B. Conrey and A. Ghosh, Mean values of the Riemann zeta-function, Mathematika 31 (1984) pp. $159-161$.

[CF] J. B. Conrey and D. W. Farmer, Mean values of L-functions and symmetry, Internat. Math. Res. Notices (2000) 17 pp. 883-908.

[CFKRS] J.B. Conrey, D.W. Farmer, J. Keating, M. Rubinstein, and N.C. Snaith, Integral moments of L-functions, Proc. Lond. Math. Soc. 91 (2005) 33104, arxiv.org/abs/math.NT/0206018

[CKRS] J.B. Conrey, J. Keating, M. Rubinstein, and N.C. Snaith, On the frequency of vanishing of quadratic twists of modular L-functions. in "Number Theory for the Millennium I"; MA Bennett et al., eds, A.K. Peters, Natick, 2002. math.NT/0012043

[CFZ] J. B. Conrey, D. W. Farmer, and M. R. Zirnbauer, Howe pairs, supersymmetry, and ratios of random characteristic polynomials for the classical compact groups, preprint

[CS] J.B. Conrey and N.C. Snaith, Applications of the L-functions ratios conjectures, preprint math.NT/0509480

[D] E. Dueñez, Random matrix ensembles associated to compact symmetric spaces, Commun. Math. Phys, 244(1), 29-61, 2004. math-ph/0111005

$[\mathrm{H}] \quad \mathrm{H}$. Helfgott, On the distribution of root numbers in families of elliptic curves, preprint, math.NT/0408141

[ILS] H. Iwaniec, W. Luo, and P. Sarnak, Low lying zeros of families of L-functions, Inst. Hautes Etudes Sci. Publ. Math. No. 91 (2000), 55131 (2001).

[IS] H. Iwaniec and P. Sarnak, The non-vanishing of central values of automorphic L-functions and Landau-Siegel zeros, Israel J. Math. 120 (2000), part A, 155177.

[KSa] N. M. Katz and P. Sarnak, Random matrices, Frobenius eigenvalues, and monodromy, AMS Colloquium Publications, 45 AMS, Providence, RI (1999).

[KS1] J. P. Keating and N. C. Snaith, Random matrix theory and $\zeta\left(\frac{1}{2}+i t\right)$, Comm. Math. Phys. 214 (2000) pp. 57-89.

[KS2] J. P. Keating and N. C. Snaith, Random matrix theory and L-functions at $s=\frac{1}{2}$, Comm. Math. Phys. 214 (2000) pp. 91-110.

[KMV] E. Kowalski, P. Michel, and J. VanderKam, Mollification of the fourth moment of automorphic L-functions and arithmetic applications, Invent. Math. 142 (2000), no. 1, 95151.

[M] S. J. Miller, 1- and 2-Level Densities for Families of Elliptic Curves: Evidence for the Underlying Group Symmetries, Compositio Mathematica 140 (2004), no. 4, 952-992. math.NT/0310159

[MD1] S. J. Miller, Investigations of Zeros Near the Central Point of Elliptic Curve L-Functions, with an appendix by E. Dueñez. math.NT/0508150

[MD2] S. J. Miller and E. Dueñez, The Low Lying Zeros of a GL(4) and a GL(6) family of L-functions, preprint, math.NT/0506462

[Mon] H.L. Montgomery, The pair correlation of zeros of the Riemann zeta-function, Proc. Symp. Pure Math. 24 (1973) pp. 181-93.

[Odl] A. Odlyzko, The $10^{20}$ th zero of the Riemann zeta-function and 70 million of its neighbors., Preprint (1989)

[OS1] A. Özlük and C. Snyder, Small Zeroes of Quadratic L -Functions, Bull. Aust. Math. Soc., 47, 307-319, (1993).

[OS2] A. Özlük and C. Snyder, On the distribution of the nontrivial zeros of quadratic L-functions close to the real axis, Acta Arith. 91 (1999), no. 3, 209228.

[Rub] M. Rubinstein Low-lying zeros of L-functions and random matrix theory, Duke Math. J. 109 (2001), no. 1, 147181.

[Si] J. Silverman, The average rank of an algebraic family of elliptic curves, J. reine angew. Math. $\mathbf{5 0 4}$ (1998), 227-236.

[Si2] J. Silverman, "The Arithmetic of Elliptic Curves", GTM 106, Springer-Verlag, New York, 1994. 
[Sn1] N.C. Snaith Derivatives of random matrix characteristic polynomials with applications to elliptic curves, to appear in J. Phys. A, math.NT/0508256

[Sn2] N.C. Snaith The derivative of $S O(2 N+1)$ characteristic polynomials and rank 3 elliptic curves, in this volume.

[S] Soundararajan, Nonvanishing of quadratic Dirichlet L-functions at $s=\frac{1}{2}$, Ann. of Math. (2) 152 (2000), no. 2, 447-488.

[Y1] M. Young, Lower-Order Terms of the 1-Level Density of Families of Elliptic Curves, Int. Math. Res. Not., 10 (2005), 587-633. math.NT/0408359

[Y2] M. Young, Moments of the critical values of families of elliptic curves, with applications, preprint.

[Y3] M. Young, Low-lying zeros of families of elliptic curves, to appear in J. Amer. Math. Soc., posted online September 7, 2005. math.NT/0406330

American Institute of Mathematics

360 Portage Ave.

Palo Alto, CA 94306

FARMER@AIMATH.ORG 